Conference Report

\title{
Directed Flow in Heavy-Ion Collisions and Its Implications for Astrophysics
}

\author{
Yuri B. Ivanov $1,2,3$ \\ 1 National Research Centre (NRC) “Kurchatov Institute”, Moscow 123182, Russia; y.b.ivanov@yandex.ru \\ 2 National Research Nuclear University "MEPhI", Moscow Engineering Physics Institute, \\ Moscow 115409, Russia \\ 3 Joint Institute for Nuclear Research (JINR), Dubna 141980, Russia
}

Received: 17 October 2017; Accepted: 7 November 2017; Published: 14 November 2017

\begin{abstract}
Analysis of directed flow $\left(v_{1}\right)$ of protons, antiprotons and pions in heavy-ion collisions is performed in the range of collision energies $\sqrt{s_{N N}}=2.7-39 \mathrm{GeV}$. Simulations have been done within a three-fluid model employing a purely hadronic equation of state (EoS) and two versions of the EoS with deconfinement transitions: a first-order phase transition and a smooth crossover transition. The crossover EoS is unambiguously preferable for the description of experimental data at lower collision energies $\sqrt{s_{N N}} \lesssim 20 \mathrm{GeV}$. However, at higher collision energies $\sqrt{s_{N N}} \gtrsim 20 \mathrm{GeV}$, the purely hadronic EoS again becomes advantageous. This indicates that the deconfinement EoS in the quark-gluon sector should be stiffer at high baryon densities than those used in the calculation. The latter finding is in agreement with that discussed in astrophysics in connection with existence of hybrid stars with masses up to about two solar masses.
\end{abstract}

Keywords: heavy-ion collisions; directed flow; hydrodynamics; deconfinement; hybrid stars

PACS: $25.75 .-q ; 25.75 . \mathrm{Nq} ; 24.10 . \mathrm{Nz}$

\section{Introduction}

The directed flow [1] is one of the key observables in heavy ion collisions. Nowadays, it is defined as the first coefficient, $v_{1}$, in the Fourier expansion of a particle distribution, $d^{2} N / d y d \phi$, in azimuthal angle $\phi$ with respect to the reaction plane [2,3]

$$
\frac{d^{2} N}{d y d \phi}=\frac{d N}{d y}\left(1+\sum_{n=1}^{\infty} 2 v_{n}(y) \cos (n \phi)\right),
$$

where $y$ is the longitudinal rapidity of a particle. The directed flow is mainly formed at an early (compression) stage of the collisions and hence is sensitive to early pressure gradients in the evolving nuclear matter $[4,5]$. As the EoS is harder, stronger pressure is developed. Thus, the directed flow probes the stiffness of the nuclear EoS at the early stage of nuclear collisions [6], which is of prime interest for heavy-ion research and astrophysics.

In Refs. [7-9], a significant reduction of the directed flow in the first-order phase transition to the quark-gluon phase (QGP) (the so-called "softest-point" effect) was predicted, which results from decreasing the pressure gradients in the mixed phase as compared to those in pure hadronic and quark-gluon phases. It was further predicted [10-12] that the directed flow as a function of rapidity exhibits a wiggle near the midrapidity with a negative slope near the midrapidity, when the incident energy is in the range corresponding to onset of the first-order phase transition. Thus, the wiggle near the midrapidity and the wiggle-like behavior of the excitation function of the midrapidity $v_{1}$ slope were put forward as a signature of the QGP phase transition. In Ref. [13], it was found that the QGP 
EoS is not a necessarily prerequisite for occurrence of the midrapidity $v_{1}$ wiggle: A certain combination of space-momentum correlations may result in a negative slope in the rapidity dependence of the directed flow in high-energy nucleus-nucleus collisions. However, this mechanism can be realized only when colliding nuclei become quite transparent so that they pass through each other at the early stage of the collision.

The directed flow of identified hadrons-protons, antiprotons, positive and negative pions-in $\mathrm{Au}+\mathrm{Au}$ collisions was recently measured in the energy range $\sqrt{s_{N N}}=(7.7-39) \mathrm{GeV}$ by the STAR collaboration within the framework of the beam energy scan (BES) program at the BNL Relativistic Heavy Ion Collider (RHIC) [14]. These data have been already discussed in Refs. [15-22]. The Frankfurt group [15] did not succeed to describe the data and to obtain conclusive results. Within a hybrid approach [23], the authors found that there is no sensitivity of the directed flow on the EoS and, in particular, on the occurrence of a first-order phase transition. One of the possible reasons of this result can be that the initial stage of the collision in all scenarios is described within the Ultrarelativistic Quantum Molecular Dynamics (UrQMD) [24] in the hybrid approach. However, this initial stage does not solely determine the final directed flow because the UrQMD results still differ from those obtained within the hybrid approach [23].

In Refs. [16-18], the new STAR data were analyzed within two complementary approaches: kinetic transport approaches of the parton-hadron string dynamics (PHSD) [25] and its purely hadronic version (HSD) [26]), and a hydrodynamic approach of the relativistic three-fluid dynamics (3FD) [27,28]. In contrast to other observables, the directed flow was found to be very sensitive to the accuracy settings of the numerical scheme. Accurate calculations require a very high memory and computation time.

In the present contribution, we refine conclusions on the relevance of used EoSs, in particular, on the stiffness of the EoS at high baryon densities in the QGP sector based on the analysis performed in Refs. [16-18].

\section{The 3FD Model}

The 3FD approximation is a minimal way to simulate the early-stage nonequilibrium in the colliding nuclei at high incident energies. The 3FD model [27] describes a nuclear collision from the stage of the incident cold nuclei approaching each other, to the final freeze-out stage. Contrary to the conventional one-fluid dynamics, where a local instantaneous stopping of matter of the colliding nuclei is assumed, the 3FD considers inter-penetrating counter-streaming flows of leading baryon-rich matter, which gradually decelerate each other due to mutual friction. The basic idea of a 3FD approximation to heavy-ion collisions is that a generally nonequilibrium distribution of baryon-rich matter at each space-time point can be represented as a sum of two distinct contributions initially associated with constituent nucleons of the projectile and target nuclei. In addition, newly produced particles, populating predominantly the midrapidity region, are attributed to a third, so-called fireball fluid that is governed by the net-baryon-free sector of the EoS.

At the final stage of the collision, the $\mathrm{p}$ - and $\mathrm{t}$-fluids are either spatially separated or mutually stopped and unified, while the f-fluid, predominantly located in the midrapidity region, keeps its identity and still overlaps with the baryon-rich fluids to a lesser (at high energies) or greater (at lower energies) extent. The freeze-out is performed accordingly to the procedure described in Ref. [27] and in more detail in Refs. [29,30].

Different EoSs can be implemented in the 3FD model. A key point is that the 3FD model is able to treat a deconfinement transition at the early nonequilibrium stage of the collision, when the directed flow is mainly formed. In this work, we apply a purely hadronic EoS [31], an EoS with a crossover transition as constructed in Ref. [32] and an EoS with a first-order phase transition into the QGP [32]. These are illustrated in Figure 1. Note that an onset of deconfinement in the two-phase EoS takes place at rather high baryon densities, above $n \sim 8 n_{0}$. In EoSs compatible with constraints on the occurrence of the quark matter phase in massive neutron stars, the phase coexistence starts at about $4 n_{0}$ [33]. An example of such an EoS, the DD2 EoS [34], is also displayed in Figure 1. The DD2 EoS 
will be discussed below. As it will be argued below, this excessive softness of the deconfinement EoSs of Ref. [32] is an obstacle for proper reproduction of the directed flow at high collision energies.

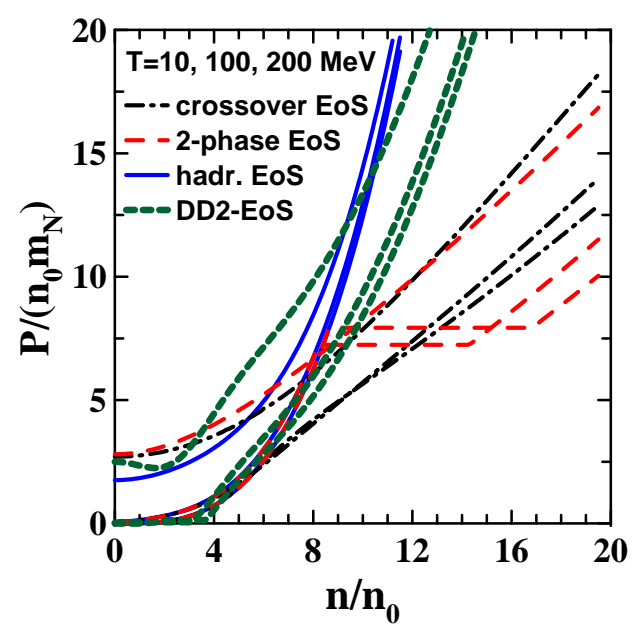

Figure 1. Pressure scaled by the product of normal nuclear density $\left(n_{0}=0.15 \mathrm{fm}^{-3}\right)$ and nucleon mass $\left(m_{N}\right)$ versus baryon density (scaled by $n_{0}$ ) for three EoSs used in the simulations and also for the DD2 EoS [34] that is compatible with astrophysical constraints. Results are presented for three different temperatures $T=10,100$ and $200 \mathrm{MeV}$ (bottom-up for corresponding curves).

In recent papers [28,35-40] a large variety of bulk observables has been analyzed with these three EoSs: the baryon stopping $[28,37]$, yields of different hadrons, their rapidity and transverse momentum distributions [35,36,38], the elliptic flow of various species [39,40]. This analysis has been done in the same range of incident energies as that in the present paper. Comparison with available data indicated a definite advantage of the deconfinement scenarios over the purely hadronic one especially at high collision energies. The physical input of the present 3FD calculations is described in detail in Ref. [28].

\section{Results}

The 3FD simulations were performed for mid-central $\mathrm{Au}+\mathrm{Au}$ collisions, i.e., at impact parameter $b=6 \mathrm{fm}$. Following the experimental conditions, the acceptance $p_{T}<2 \mathrm{GeV} / \mathrm{c}$ for transverse momentum $\left(p_{T}\right)$ of the produced particles is applied to all considered hadrons. This choice is commented in Ref. [18]. In the 3FD model, particles are not isotopically distinguished; i.e., the model deals with nucleons, pions, etc. rather than with protons, neutrons, $\pi^{+}, \pi^{-}$and $\pi^{0}$. Therefore, the $v_{1}$ values of protons, antiprotons and pions presented below, in fact, are $v_{1}$ of nucleons, antinucleons and all (i.e., $\pi^{+}, \pi^{-}$and $\pi^{0}$ ) pions. The directed flow $v_{1}(y)$ as a function of rapidity $y$ at BES-RHIC bombarding energies is presented in Figure 2 for pions, protons and antiprotons.

As seen, the first-order-transition scenario gives results for the proton $v_{1}$, which strongly differ from those in the crossover scenario at $\sqrt{s_{N N}}=7.7$ and $19.6 \mathrm{GeV}$. This is in contrast to other bulk observables analyzed so far $[28,35-40]$. At $\sqrt{s_{N N}}=39 \mathrm{GeV}$, the directed flow of all considered species practically coincides within the first-order-transition and crossover scenarios. It means that the crossover transition to the QGP has been practically completed at $\sqrt{s_{N N}}=39 \mathrm{GeV}$. It also suggests that the region $7.7 \leq \sqrt{s_{N N}} \leq 30 \mathrm{GeV}$ is the region of the crossover transition.

The crossover EoS is definitely the best in reproduction of the proton $v_{1}(y)$ at $\sqrt{s_{N N}} \leq 20 \mathrm{GeV}$. However, surprisingly, the hadronic scenario becomes preferable for the proton $v_{1}(y)$ at $\sqrt{s_{N N}}>20 \mathrm{GeV}$. A similar situation takes place in the PHSD/HSD transport approach. Indeed, predictions of the HSD model (i.e., without the deconfinement transition) for the proton $v_{1}(y)$ become preferable at $\sqrt{s_{N N}}>30 \mathrm{GeV}$ [16], i.e., at somewhat higher energies than in the 3FD model. Moreover, the proton $v_{1}$ predicted by the UrQMD model, as cited in the experimental paper [14], and, in the recent theoretical work [15], better reproduces the proton $v_{1}(y)$ data at high collision energies than 
the PHSD and 3FD-deconfinement models do. Note that the UrQMD model is based on the hadronic dynamics. All these observations could be considered as evidence of a problem in the QGP sector of a EoS. At the same time, the antiproton directed flow at $\sqrt{s_{N N}}>10 \mathrm{GeV}$ definitely indicates a preference of the crossover scenario within both the PHSD/HSD and 3FD approaches.

This puzzle has a natural resolution within the 3FD model. The QGP sector of the EoSs with deconfinement [32] was fitted to the lattice QCD data at zero net-baryon density and just extrapolated to nonzero baryon densities. The protons mainly originate from baryon-rich fluids that are governed by the EoS at finite baryon densities. The too strong proton antiflow within the crossover scenario at $\sqrt{s_{N N}}>20 \mathrm{GeV}$ is a sign of too soft QGP EoS at high baryon densities. In general, the antiflow or a weak flow indicates softness of an EoS [6-13]. Predictions of the first-order-transition EoS, the QGP sector of which is constructed in the same way as that of the crossover one, fail even at lower collision energies, when the QGP starts to dominate in the collision dynamics, i.e., at $\sqrt{s_{N N}} \gtrsim 15 \mathrm{GeV}$. This fact also supports the conjecture on a too soft QGP sector at high baryon densities in the used EoSs.

At the same time, the net-baryon-free (fireball) fluid is governed by the EoS at zero net-baryon density. This fluid is a main source of antiprotons (more than $80 \%$ near midrapidity at $\sqrt{s_{N N}}>20 \mathrm{GeV}$ and $b=6 \mathrm{fm}), v_{1}(y)$ of which is in good agreement with the data at $\sqrt{s_{N N}}>20 \mathrm{GeV}$ within the crossover scenario and even in perfect agreement-within the first-order-transition scenario at $\sqrt{s_{N N}}=39 \mathrm{GeV}$. It is encouraging because at zero net-baryon density the QGP sector of the EoSs is fitted to the lattice QCD data and therefore can be trusted. The crossover scenario, as well as all other scenarios, definitely fails to reproduce the antiproton $v_{1}(y)$ data at $7.7 \mathrm{GeV}$. The reason is low multiplicity of produced antiprotons. The antiproton multiplicity in the mid-central $(b=6 \mathrm{fm})$ $\mathrm{Au}+\mathrm{Au}$ collision at $7.7 \mathrm{Gev}$ is 1 within the deconfinement scenarios and 3 within the hadronic scenario. Therefore, the hydrodynamical approach based on the grand canonical ensemble is certainly inapplicable to the antiprotons in this case. The grand canonical ensemble, with respect to conservation laws, gives a satisfactory description of abundant particle production in heavy ion collisions. However, when applying the statistical treatment to rare probes, one needs to treat the conservation laws exactly, which is the canonical approach. The exact conservation of quantum numbers is known to reduce the phase space available for particle production due to additional constraints appearing through requirements of local quantum number conservation. An example of applying the canonical approach to the strangeness production can be found in [41] and references therein.

The pions are produced from all fluids: near midrapidity $\sim 60 \%$ from the baryon-rich fluids and $\sim 40 \%$ from the net-baryon-free one at $\sqrt{s_{N N}}>20 \mathrm{GeV}$. Hence, the disagreement of the pion $v_{1}$ with data, resulting from redundant softness of the QGP EoS at high baryon densities, is moderate at $\sqrt{s_{N N}}>20 \mathrm{GeV}$. In general, the pion $v_{1}$ is less sensitive to the EoS as compared to the proton and antiproton ones. As seen from Figure 2, the deconfinement scenarios are definitely preferable for the pion $v_{1}(y)$ at $\sqrt{s_{N N}}<20 \mathrm{GeV}$. Though, the hadronic-scenario results are not too far from the experimental data. At $\sqrt{s_{N N}}=39 \mathrm{GeV}$, the hadronic scenario gives even the best description of the pion data because of a higher stiffness of the hadronic EoS at high baryon densities, as compared with that in the considered versions of the QGP EoS.

Thus, all of the analyzed data testify in favor of a harder QGP EoS at high baryon densities than those used in the simulations, i.e., the desired QGP EoS should be closer to the used hadronic EoS at the same baryon densities (see Figure 1). At the same time, a moderate softening of the QGP EoS at moderately high baryon densities is agreement with data at $7.7 \lesssim \sqrt{s_{N N}} \lesssim 20 \mathrm{GeV}$.

Here, it is appropriate to mention a discussion on the QGP EoS in astrophysics. In Ref. [42], it was demonstrated that the QGP EoS can be almost indistinguishable from the hadronic EoS at high baryon densities relevant to neutron stars. In particular, this gives a possibility to explain hybrid stars with masses up to about 2 solar masses $\left(M_{\odot}\right)$, in such a way that "hybrid stars masquerade as neutron stars" [42]. The discussion of such a possibility has been revived after measurements on two binary pulsars PSR J1614-2230 [43] and PSR J03481+0432 [44] resulted in the pulsar masses of 
$(1.97 \pm 0.04) M_{\odot}$ and $(2.01 \pm 0.04) M_{\odot}$, respectively. The obtained results on the directed flow give us another indication of a required hardening of the QGP EoS at high baryon densities.

In this respect, it is instructive to compare the DD2 EoS [34], which is compatible with the existence of hybrid stars with masses up to about 2 solar masses, with those used in the present simulations (see Figure 1). As seen, the DD2 EoS is much closer to the hadronic EoS at high baryon densities as compared to the deconfinement EoSs used in the calculation. This gives hope to the better reproduction of the directed flow at high collision energies $\sqrt{s_{N N}} \gtrsim 20 \mathrm{GeV}$ with the DD2 EoS.

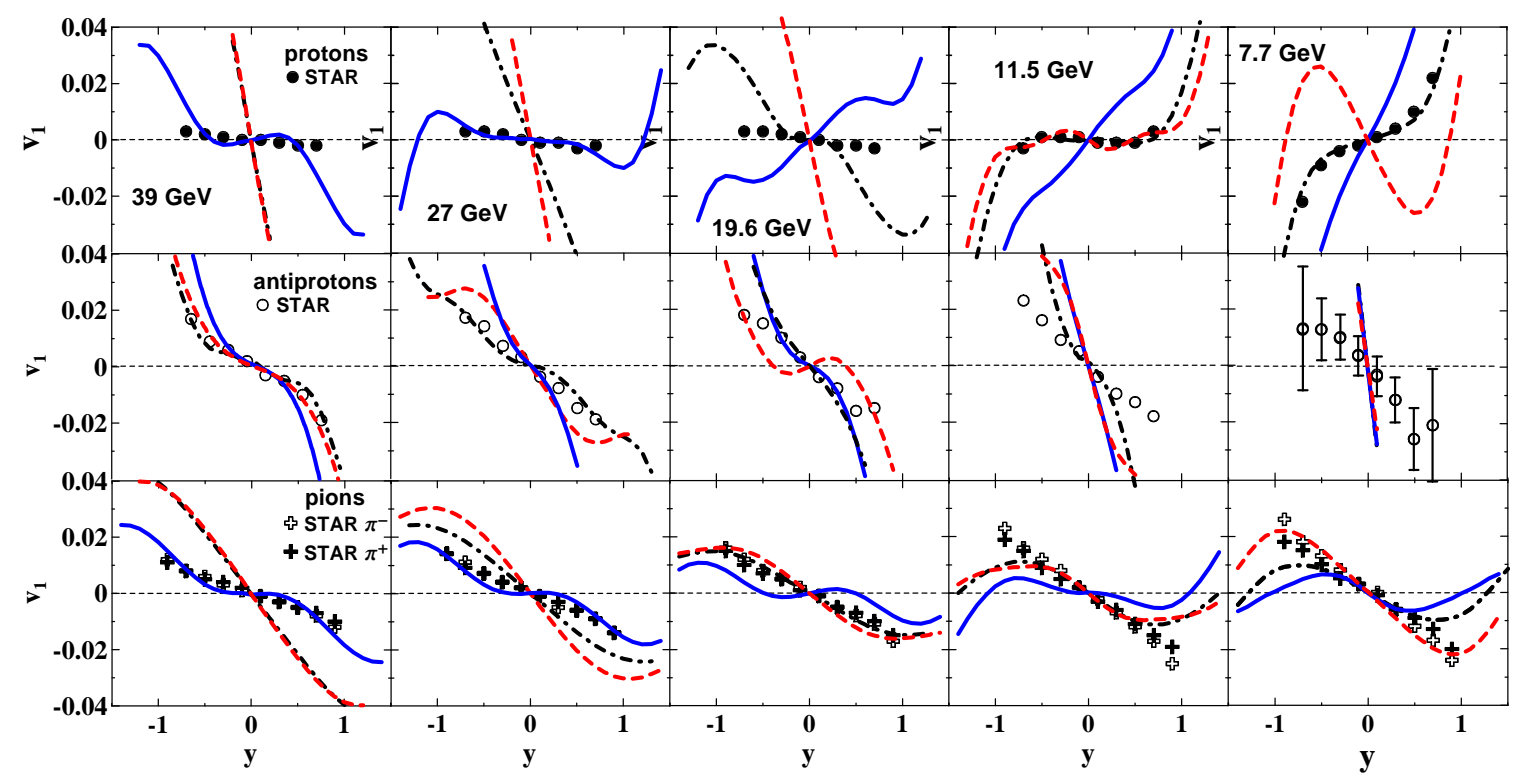

Figure 2. The directed flow $v_{1}(y)$ for protons, antiprotons and pions from mid-central $(b=6 \mathrm{fm})$ $\mathrm{Au}+\mathrm{Au}$ collisions at $\sqrt{s_{N N}}=7.7-39 \mathrm{GeV}$ calculated with different EoSs. Experimental data are from the STAR collaboration [14].

The slope of the directed flow at the midrapidity is often used to quantify variation of the directed flow with collision energy. The excitation functions for the slopes of the $v_{1}$ distributions at midrapidity are summarized in Figure 3, where earlier experimental results from the AGS [45] and SPS [46] are also presented. As noted above, the best reproduction of the data at $\sqrt{s_{N N}}<20 \mathrm{GeV}$ is achieved with the crossover EoS. The proton $d v_{1} / d y$ within the first-order-transition scenario exhibits a wiggle earlier predicted in Refs. $[8,9,11,12]$. The first-order-transition results demonstrate the worst agreement with the proton and antiproton data on $d v_{1} / d y$. The first-order-transition $d v_{1} / d y$ does not coincide with that for the crossover scenario even at high collision energies (i.e., at $10 \mathrm{GeV} \lesssim \sqrt{s_{N N}} \lesssim 30 \mathrm{GeV}$ ) because the corresponding EoSs are not identical in the region of high baryon densities where the smooth crossover transition is not completed yet, cf. Figure 1, and because of different friction terms, which were separately fitted for each EoS in order to reproduce other bulk observables.

The discrepancies between experiment and the 3FD predictions are smaller for the purely hadronic EoS, however, the agreement for the crossover EoS is definitely better, though it is far from being perfect. However, the poor reproduction of the proton $v_{1}$ slope at low energies $\left(\sqrt{s_{N N}}<5 \mathrm{GeV}\right)$, it is still questionable because the same data, but in terms of the transverse in-plane momentum, $\left\langle P_{x}\right\rangle$, are almost perfectly reproduced by the crossover scenario [17,47]. It is difficult to indicate the beginning of the crossover transition because the crossover results become preferable beginning with relatively low collision energies $\left(\sqrt{s_{N N}}>3 \mathrm{GeV}\right)$. However, the beginning of the crossover transition can be approximately pointed out as $\sqrt{s_{N N}} \simeq 4 \mathrm{GeV}$.

The above discussed problems of the crossover scenario reveal themselves also in the $d v_{1} / d y$ plot. At high energies $\left(\sqrt{s_{N N}}>20 \mathrm{GeV}\right)$, the slopes also indicate that the used deconfinement EoSs in the quark-gluon sector at zero baryon chemical potential are quite suitable for reproduction of the 
antiproton $d v_{1} / d y$ while those at high baryon densities (proton slope) should be stiffer in order to achieve better description of proton $d v_{1} / d y$. A combined effect of this excessive softness of the QGP EoS and the reducing baryon stopping results in more and more negative proton slopes at high collision energies. This is in line with the mechanism discussed in Ref. [13]. The pion flow partially follows the proton pattern, as discussed above. Therefore, the pion $v_{1}$ slope also becomes more negative with energy rise.

Of course, the 3FD model does not include all factors determining the directed flow. Initial-state fluctuations, which in particular make the directed flow to be nonzero even at midrapidity, are out of the scope of the 3FD approach. Apparently, these fluctuations can essentially affect the directed flow at high collision energies, when the experimental flow itself is very weak. Another point is so-called afterburner, i.e., the kinetic evolution after the the hydrodynamical freeze-out. This stage is absent in the conventional version of the 3FD. Recently, an event generator THESEUS based on the output of the 3FD model was constructed [48]. Thus, constructed output of the 3FD model can be further evolved within the UrQMD model. Results of Ref. [48] show that such kind of the afterburner mainly affects the pion $v_{1}$ at peripheral rapidities and makes it more close to the STAR data [14]. At $\sqrt{s_{N N}}<5 \mathrm{GeV}$, the midrapidity region of the pion $v_{1}$ is also affected; however, the pion data are absent at these energies. An additional source of uncertainty is the freeze-out. In Ref. [15], it was demonstrated that the freeze-out procedure and, in particular, its criterion also strongly affect the directed flow. Different freeze-out procedures were not tested within the 3FD model because such a test would amount to the analysis of all other bulk observables that can also be affected by the freeze-out change [29]. Such an extensive test would imply a huge amount of computations. However, this source of uncertainty should be mentioned.
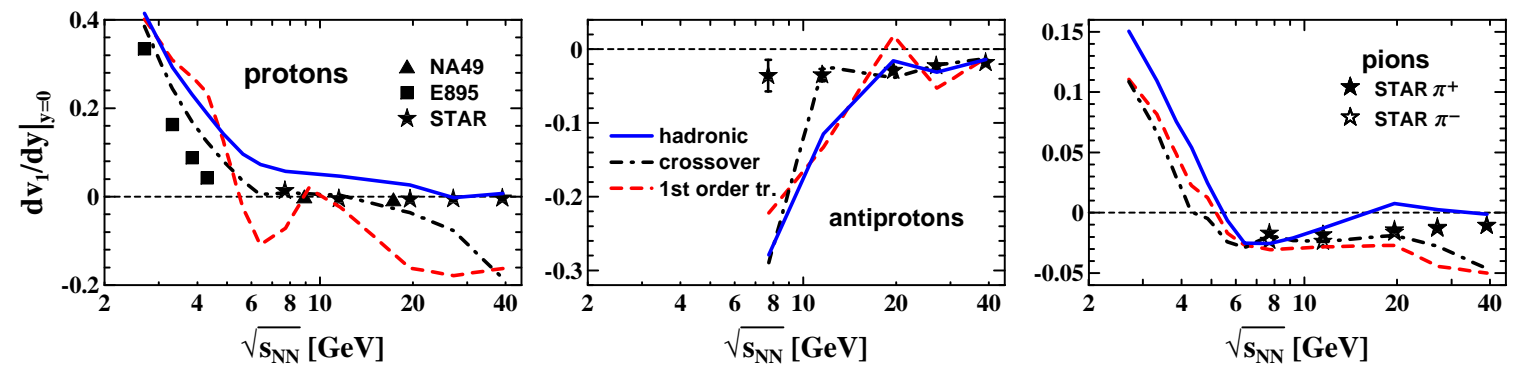

Figure 3. The beam energy dependence of the directed flow slope at midrapidity for protons, antiprotons and pions from mid-central $(b=6 \mathrm{fm}) \mathrm{Au}+\mathrm{Au}$ collisions calculated with different EoSs. The experimental data are from Refs. [14,45,46].

\section{Conclusions}

In conclusion, the crossover EoS is unambiguously preferable for the most part of experimental data in the considered energy range, though this description is not perfect. Based on the crossover EoS of Ref. [32], the directed flow in semi-central $\mathrm{Au}+\mathrm{Au}$ collisions indicates that the crossover deconfinement transition takes place in the wide range incident energies $4 \lesssim \sqrt{s_{N N}} \lesssim 30 \mathrm{GeV}$. In part, this wide range could be a consequence of that the crossover transition constructed in Ref. [32] is very smooth. In this respect, this version of the crossover EoS certainly contradicts results of the lattice QCD calculations, where a fast crossover, at least at zero chemical potential, was found [49].

At highest computed energies of $\sqrt{s_{N N}}>20 \mathrm{GeV}$, the obtained results indicate that the deconfinement EoSs in the QGP sector should be stiffer at high baryon densities than those used in the calculation, i.e., more similar to the purely hadronic EoS. This observation is in agreement with that discussed in astrophysics, in particular, in connection with a possibility to explain hybrid stars with masses up to about two solar masses. The constraint of existence of such hybrid stars results in the requirement of quite stiff QGP EoS at high baryon densities that is very similar to the hadronic EoS. The obtained results on the directed flow give us another indication of a required 
hardening of the QGP EoS at high baryon densities. However, this is only an indirect similarity with the astrophysical conjecture because directed-flow simulations are sensitive to the EoS at high temperatures $(T>100 \mathrm{MeV})$ while the hybrid-star calculations are based on zero-temperature EoS.

Acknowledgments: Fruitful discussions with David Blaschke, Hermann Wolter and Dmitri N. Voskresensky are gratefully acknowledged. This work was carried out using computing resources of the federal collective usage center "Complex for simulation and data processing for mega-science facilities" at NRC "Kurchatov Institute", http:/ /ckp.nrcki.ru/. This work was supported by the Russian Science Foundation, Grant No. 17-12-01427.

Conflicts of Interest: The author declares no conflict of interest.

\section{References}

1. Danielewicz, P.; Odyniec, G. Transverse Momentum Analysis of Collective Motion in Relativistic Nuclear Collisions. Phys. Lett. B 1985, 157, 146-150.

2. Voloshin, S.; Zhang, Y. Flow study in relativistic nuclear collisions by Fourier expansion of Azimuthal particle distributions. Z. Phys. C 1996, 70, 665-671.

3. Voloshin, S.A.; Poskanzer, A.M.; Snellings, R. Collective phenomena in non-central nuclear collisions. In Relativistic Heavy Ion Physics; Landolt-Börnstein-Group I Elementary Particles, Nuclei and Atoms; Springer-Verlag: New York, NY, USA, 2010.

4. Sorge, H. Elliptical flow: A signature for early pressure in ultrarelativistic nucleus-nucleus collisions. Phys. Rev. Lett. 1997, 78, 2309-2312.

5. Herrmann, N.; Wessels, J.P.; Wienold, T. Collective flow in heavy ion collisions. Ann. Rev. Nucl. Part. Sci. $1999,49,581-632$.

6. Russkikh, V.N.; Ivanov, Y.B. Collective flow in heavy-ion collisions for Elab $=1 \times 160 \mathrm{GeV} / \mathrm{nucleon}$. Phys. Rev. C 2006, 74, 034904.

7. Hung, C.M.; Shuryak, E.V. Hydrodynamics near the QCD phase transition: Looking for the longest lived fireball. Phys. Rev. Lett. 1995, 75, 4003-4006.

8. Rischke, D.H.; Pursun, Y.; Maruhn, J.A.; Stöcker, H.; Greiner, W. The phase transition to the quark-gluon plasma and its effects on hydrodynamic flow. Heavy Ion Phys. 1995, 1, 309-322.

9. Rischke, D.H. Hydrodynamics and collective behaviour in relativistic nuclear collisions. Nucl. Phys. A 1996, 610, 88-101.

10. Csernai, L.P.; Rohrich, D. Third flow component as QGP signal. Phys. Lett. B 1999, 458, 454-459.

11. Brachmann, J.; Soff, S.; Dumitru, A.; Stöcker, H.; Maruhn, J.A.; Greiner, W.; Rischke, D.H. Antiflow of nucleons at the softest point of the equation of state. Phys. Rev. C 2000, 61, 024909.

12. Stöcker, H. Collective flow signals the quark-gluon plasma. Nucl. Phys. A 2005, 750, 121-147.

13. Snellings, R.J.M.; Sorge, H.; Voloshin, S.A.; Wang, F.Q.; Xu, N. Novel rapidity dependence of directed flow in high energy heavy ion collisions. Phys. Rev. Lett. 2000, 84, 2803-2805.

14. Adamczyk, L.; Adkins, J.K.; Agakishiev, G.; Aggarwal, M.M.; Ahammed, Z.; Alekseev, I.; Alford, J.; Anson, C.D.; Aparin, A.; Arkhipkin, D.; et al. Beam-Energy Dependence of Directed Flow of Protons, Antiprotons and Pions in Au+Au Collisions. Phys. Rev. Lett. 2014, 112, 162301.

15. Steinheimer, J.; Auvinen, J.; Petersen, H.; Bleicher, M.; Stöcker, H. Examination of directed flow as a signal for a phase transition in relativistic nuclear collisions. Phys. Rev. C 2014, 89, 054913.

16. Konchakovski, V.; Cassing, W.; Ivanov, Y.; Toneev, V. Examination of the directed flow puzzle in heavy-ion collisions. Phys. Rev. C 2014, 90, 014903.

17. Ivanov, Y.B.; Soldatov, A.A. Directed flow indicates a cross-over deconfinement transition in relativistic nuclear collisions. Phys. Rev. C 2015, 91, 024915.

18. Ivanov, Y.B.; Soldatov, A.A. What can we learn from the directed flow in heavy-ion collisions at BES RHIC energies? Eur. Phys. J. A 2016, 52, 10.

19. Nara, Y.; Ohnishi, A. Does non-monotonic behavior of directed flow signal the onset of deconfinement? Nucl. Phys. A 2016, 956, 284-287.

20. Nara, Y.; Niemi, H.; Ohnishi, A.; Stöcker, H. Examination of directed flow as a signature of the softest point of the equation of state in QCD matter. Phys. Rev. C 2016, 94, 034906.

21. Nara, Y.; Niemi, H.; Steinheimer, J.; Stöcker, H. Equation of state dependence of directed flow in a microscopic transport model. Phys. Lett. B 2017, 769, 543-548. 
22. Singha, S.; Shanmuganathan, P.; Keane, D. The first moment of azimuthal anisotropy in nuclear collisions from AGS to LHC energies. Adv. High Energy Phys. 2016, 2016, 2836989.

23. Petersen, H.; Steinheimer, J.; Burau, G.; Bleicher, M.; Stöcker, H. A Fully Integrated Transport Approach to Heavy Ion Reactions with an Intermediate Hydrodynamic Stage. Phys. Rev. C 2008, 78, 044901.

24. Bass, S.; Belkacem, M.; Bleicher, M.; Brandstetter, M.; Bravina, L.; Ernst, C.; Gerland, L.; Hofmann, M.; Hofmann, S.; Konopka, J.; et al. Progress in Particle and Nuclear Physics. Prog. Part. Nucl. Phys. 1998, 41, 255-369.

25. Cassing, W.; Bratkovskaya, E.L. Parton-hadron-string dynamics: An off-shell transport approach for relativistic energies. Nucl. Phys. A 2009, 831, 215-242.

26. Cassing, W.; Bratkovskaya, E.L. Hadronic and electromagnetic probes of hot and dense nuclear matter. Phys. Rep. 1999, 308, 65-233.

27. Ivanov, Y.B.; Russkikh, V.N.; Toneev, V.D. Relativistic heavy-ion collisions within three-fluid hydrodynamics: Hadronic scenario. Phys. Rev. C 2006, 73, 044904.

28. Ivanov, Y.B. Alternative Scenarios of Relativistic Heavy-Ion Collisions: I. Baryon Stopping. Phys. Rev. C 2013, 87,064904 .

29. Russkikh, V.N.; Ivanov, Y.B. Vorticity in heavy-ion collisions at the JINR Nuclotron-based Ion Collider fAcility. Phys. Rev. C 2007, 76, 054907.

30. Ivanov, Y.B.; Russkikh, V.N. On freeze-out problem in relativistic hydrodynamics. Phys. Atomic Nucl. 2009, 72, 1238-1244.

31. Galitsky, V.M.; Mishustin, I.N. Relativistic effects in collision of heavy ions. Sov. J. Nucl. Phys. 1979, 29, 363-373. (In Russian)

32. Khvorostukhin, A.S.; Skokov, V.V.; Redlich, K.; Toneev, V.D.; Redlich, K. Lattice QCD constraints on the nuclear equation of state. Eur. Phys. J. C 2006, 48, 531-543.

33. Klähn, T.; Łastowiecki, R.; Blaschke, D.B. Implications of the measurement of pulsars with two solar masses for quark matter in compact stars and heavy-ion collisions: A Nambu Jona-Lasinio model case study. Phys. Rev. D 2013, 88, 085001.

34. Typel, S.; Ropke, G.; Klahn, T.; Blaschke, D.; Wolter, H.H. Composition and thermodynamics of nuclear matter with light clusters. Phys. Rev. C 2010, 81, 015803.

35. Ivanov, Y.B. Alternative Scenarios of Relativistic Heavy-Ion Collisions: II. Particle Production. Phys. Rev. C 2013, 87, 064905.

36. Ivanov, Y.B. Alternative Scenarios of Relativistic Heavy-Ion Collisions: III. Transverse Momentum Spectra. Phys. Rev. C 2014, 89, 024903.

37. Ivanov, Y.B. Baryon Stopping as a Probe of Deconfinement Onset in Relativistic Heavy-Ion Collisions. Phys. Lett. B 2013, 721, 123-130.

38. Ivanov, Y.B. Phase Evolution and Freeze-out within Alternative Scenarios of Relativistic Heavy-Ion Collisions. Phys. Lett. B 2013, 726, 422-426.

39. Ivanov, Y.B. Elliptic Flow of Protons and Antiprotons in Au+Au Collisions at $\sqrt{s_{N N}}=7.7-62.4 \mathrm{GeV}$ within Alternative Scenarios of Three-Fluid Dynamics. Phys. Lett. B 2013, 723, 475-480.

40. Ivanov, Y.B.; Soldatov, A.A. Elliptic Flow in Heavy-Ion Collisions at Energies $\sqrt{s_{N N}}=2.7-39 \mathrm{GeV}$. Phys. Rev. C 2015, 91, 024914.

41. Hamieh, S.; Redlich, K.; Tounsi, A. Canonical description of strangeness enhancement from $\mathrm{p}-\mathrm{A}$ to $\mathrm{Pb} \mathrm{Pb}$ collisions. Phys. Lett. B 2000, 486, 61-66.

42. Alford, M.; Braby, M.; Paris, M.W.; Reddy, S. Hybrid stars that masquerade as neutron stars. Astrophys. J. 2005, 629, 969-978.

43. Demorest, P.; Pennucci, T.; Ransom, S.; Roberts, M.; Hessels, J. Shapiro Delay Measurement of A Two Solar Mass Neutron Star. Nature 2010, 467, 1081-1083.

44. Antoniadis, J.; Freire, P.C.C.; Wex, N.; Tauris, T.M.; Lynch, R.S.; van Kerkwijk, M.H.; Kramer, M.; Bassa, C. A Massive Pulsar in a Compact Relativistic Binary. Science 2013, 340, 1233232.

45. Ajitanand, N.N.; Alexander, J.; Anderson, M.; Best, D.; Brady, F.P.; Case, T.; Caskey, W.; Cebra, D.; Chance, J.; Cole, B. Sideward Flow in Au+Au Collisions between 2A and 8A GeV. Phys. Rev. Lett. 2000, 84, 5488.

46. Alt, C.; Anticic, T.; Baatar, B.; Barna, D.; Bartke, J.; Behler, M.; Betev, L.; Bialkowska, H.; Billmeier, A.; Blume, $\mathrm{C}$; ; et al. Directed and elliptic flow of charged pions and protons in $\mathrm{Pb}+\mathrm{Pb}$ collisions at $40 \mathrm{~A}$ and 158 A GeV. Phys. Rev. C 2003, 68, 034903. 
47. Ivanov, Y.B.; Soldatov, A.A. Directed flow is a sensitive probe of deconfinement transition. Eur. Phys. J. A 2016, 52, 246.

48. Batyuk, P.; Blaschke, D.; Bleicher, M.; Ivanov, Y.B.; Karpenko, I.; Merts, S.; Nahrgang, M.; Petersen, H.; Rogachevsky, O. Event simulation based on three-fluid hydrodynamics for collisions at energies available at the Dubna Nuclotron-based Ion Collider Facility and at the Facility for Antiproton and Ion Research in Darmstadt. Phys. Rev. C 2016, 94, 044917.

49. Aoki, Y.; Endrodi, G.; Fodor, Z.; Katz, S.D.; Szabo, K.K. The Order of the quantum chromodynamics transition predicted by the standard model of particle physics. Nature 2006, 443, 675-678.

(C) 2017 by the author. Licensee MDPI, Basel, Switzerland. This article is an open access article distributed under the terms and conditions of the Creative Commons Attribution (CC BY) license (http:/ / creativecommons.org/licenses/by/4.0/). 